\title{
Relative numerousness judgments by squirrel monkeys
}

\author{
ROGER K. THOMAS and LAURIE CHASE \\ University of Georgia, Athens, Georgia 30602
}

\begin{abstract}
With nonnumerousness dimensions (e.g., pattern, area) controlled, three cards with two to seven black-filled circles were presented on each trial. If the center of three conditional cue lights was illuminated, the monkey was reinforced for selecting the card with the fewest circles; if two lights were on, the card with the intermediate number of circles was correct; and if three lights were on, the card with the most circles was correct. Training began with one conditional cue light and proceeded to the threelight condition. Then the one and threelight conditions were presented randomly and concurrently, followed by the two-light condition. Finally, one, two, or three lights were presented randomly and concurrently. Only one monkey met criterion on all training stages, but another monkey succeeded also through the intermediatenumber condition. It was concluded that the squirrel monkey is capable of relative numerousness judgments, including ordinal numerousness judgments. Additional discussion was concerned with the hypothesis suggested by Brown, Lenneberg, and Ettlinger (1978) that the ability to use quantitative concepts is a prerequisite to the acquisition of language.
\end{abstract}

The study of numerousness judgments by animals has long been of interest, but the experimental procedures used in most studies raise questions regarding the animals' use of number cues (see Salman, 1943; Swenson, 1970; Thomas, Fowlkes, \& Vickery, in press; Wesley, 1961, for their examinations of the literature). The more convincing studies appear to have been done with nonhuman primates, specifically Macaca mulatta (Hicks, 1956), chimpanzee (species not indicated) (Dooley \& Gill, 1977; Hayes \& Nissen, 1971), and Saimiri sciureus (Thomas et al., in press).

Using terms as defined by Thomas and Crosby (1977), the studies by Hayes and Nissen (1971), Hicks (1956), and Thomas et al. (in press) showed the animals' use of numerousness as absolute class concepts. That is, the animals learned to respond to stimuli representing "threeness," "sevenness," and so on, independently of the other stimulus choices. On the other hand, Dooley and Gill (1977) reinforced their chimpanzee for responding to numerousness on a relative basis (i.e., "fewest" vs. "most") or, in other words, as relative class concepts. Dooley and Gill used one of two conditional cues on each trial to indicate to the chimpanzee whether it should respond to the stimulus set with the fewest or the most elements.

Brown, Lenneberg, and Ettlinger (1978) compared children, chimpanzees, and rhesus monkeys on the "symbolic" use of the concepts "all," "some," "one." and "none." Specifically, the colors of the stimulus objects served as conditional cues (or symbols) to indicate which reinforcement contingency was in effect on a particular trial. If, for example, the objects were yellow, the "some" concept was supposed to be used, and the correct thing to do was to respond to more than one but not more than $\mathrm{N}-1$ objects. Compared with their performances on the "all," "one," and "none" concepts, as well as compared with the children's performances, the chimpanzees and monkeys were deficient on the "some" concept.

The present study may be compared to the Brown et al. (1978) and Dooley and Gill (1977) studies, in that conditional (or symbolic) cues were used to indicate the correct numerousness category on a given trial. Specifically, here, from one to three cue lights were illuminated on each trial. If one light was on, the monkey was reinforced for responding to a stimulus card representing the fewest elements; if two lights were on, it was reinforced for responding to the card representing the intermediate number of elements; if three lights were on, the card with the most elements was correct. The present study differed from the Dooley and Gill study primarily in that it included the intermediate number category, and it differed from that of Brown et al. primarily in that Brown et al. required a sequence of responses that was congruent with the quantitative category being presented on a trial. The present study required only that the correct quantitative judgment be followed by a single response to the stimulus card that was consistent with that judgment.

\section{METHOD}

Subjects

Three wild-born adult male squirrel monkeys (Saimiri sciureus) were used. Two (78-I-1 and 78-I-2) were used in a previous study involving absolute (as opposed to relative) numerousness judgments (Thomas et al., in press), and one (S 14) had been a subject in a conceptual volume judgment study (Thomas \& Ingram, 1979). They were housed in individual cages in a temperature- $\left(24^{\circ}-27^{\circ} \mathrm{C}\right)$ and humidity- $(50 \%-70 \%)$ controlled colony room. Timers controlled light onset at 8:00 a.m. and offset at 8:00 p.m. local time, and all testing was 
done during the light phase. The monkeys received their usual diet of Purina (25\% protein) or Wayne (24\% protein) monkey food, which was supplemented regularly with fresh fruit. Water was always available.

\section{Apparatus, General Procedure, and Pretraining}

A modified Wisconsin General Test Apparatus (WGTA) was used. The WGTA has a gray, wooden stimulus tray that has area dimensions of $27 \times 35 \mathrm{~cm}$. Three transparent, acrylic, vertically oriented card holders $(9 \times 9 \mathrm{~cm})$ with $4.5 \times 9 \mathrm{~cm}$ bases were used. Each holder was mounted flush with the front $(35 \mathrm{~cm})$ edge of the stimulus tray, two were also flush with one of the sides of the tray, and the third was centered on the front edge equidistant between the sides. The bases of the card holders, which were rendered opaque, covered food wells $(1.5 \mathrm{~cm}$ in diameter) centered beneath the base of each card holder. The card holders were attached to the stimulus tray such that a slight push by the monkey would swivel it aside, revealing the food well beneath. Attached to the WGTA, facing the monkey and immediately below the numerousness stimuli, was a row of three neon panel lamps, $2.5 \mathrm{~cm}$ apart, which served as the conditional cues.

Other than these lamps, the only illumination was provided by a $25-\mathrm{W}$ bulb mounted in the top center of the WGTA. The monkey was tested in the room in which it was housed by moving its home cage to an empty slot on the cage rack that directly approximated the WGTA. Screens prevented the other monkeys from observing the testing. To set the stimuli, the door between the monkey and the experimenter was closed. Then the experimenter's door was closed, the monkey's door was raised, and the tray was advanced slightly but not within the monkey's reach. After a 5-sec delay, the tray was advanced within reach, and the monkey was allowed $30 \mathrm{sec}$ in which to respond. Following a response, the monkey's door was closed, and the stimuli were set for the next trial. Approximately 30-60 sec elapsed between trials. In the event of an incorrect response, the trial was repeated as though it were a new trial until a correct choice was made. Only the initial error was recorded.

Two kinds of pretraining procedures were used. The first, which consisted of three stages, had no direct relationship to numerousness and will be described here as P1-P3. The second kind of pretraining, which also consisted of three stages, was related to numerousness and was interspersed among the 10 training stages; it will be described in the following section as P4-P6.

P1. A card holder, bearing a card with a black-filled triangle, was slightly displaced, allowing a currant in the food well beneath to be seen. The two remaining food wells bore card holders with blank white cards. In random order, each food well was baited and denoted by the partially displaced, triangle-bearing card holder, until the monkey had retrieved 10 currants from each food well.

P2. P2 was the same as P1, except the food wells were completely covered by the card holders.

P3. In randomly determined order, the three card holders bore the same black-filled triangle, a circle, or a square. Responses to the card bearing the triangle were reinforced. Forty-five trials per day were given until the monkey had $41 / 45$ correct and a significant $(\mathrm{p}<.01)$ "run" of successive correct responses was seen. (See Grant, 1947, and Thomas and Crosby, 1977, for details of the "runs" analysis.)

\section{Numerousness Pretraining and Training}

As indicated above, there were 10 stages of training and three stages of numerousness pretraining. These will be described below in the order in which they occurred. Pretraining will be denoted as P4-P6, and training as T1-T10.

The stimuli consisted of black-filled circles on plain white index cards that had been trimmed $(8.5 \times 8.5 \mathrm{~cm})$ to fit the card holders. Three sizes of black-filled circles were used: $2.5-\mathrm{mm}, 5.0-\mathrm{mm}$, and $10-\mathrm{mm}$ radii, or $19.6-\mathrm{mm}^{2}, 78.5-\mathrm{mm}^{2}$, and $314-\mathrm{mm}^{2}$ respectively. A 16 -point $(4$ by 4$)$ grid was used to determine the loci of the circles on the cards. The sizes of circles to be used on a card were determined randomly. The loci of the circles on the grid were determined randomly, except that each circle had to be adjacent to at least one other circle in a row, in a column, or on a diagonal; this restriction was intended to prevent clusters of circles that might give the appearance of two numerousness subsets on a card (e.g., two in one corner and three in the diagonally opposite corner). Twentyfive cards were constructed for each number. All four orientations were used; hence each number was represented by 100 distinguishable patterns. Cards representing the numbers 2-7 were used in this experiment.

Forty-five trials were given in each session. Unless otherwise specified, the same double criterion stated in P3 above was used.

P4. Randomly selected examples of the numbers 2,4 , and 6 were used on each trial, and their positions in the card holders were determined randomly. The center light in the row of panel lights was continuously illuminated. Responses to the card representing the number 2 were reinforced. It may be noted that "center light on" was a consistent cue here and that in succeeding stages responses to the card representing the fewest circles would be reinforced.

T1. Using the entire population of stimulus cards, the cards for a given trial were selected randomly, except that no successive numbers were used and three different numbers were selected (e.g., 2-4-6, 3-5-7, 2-5-7, etc.) The center panel light was on and responses to the fewest circles were reinforced.

T2. T2 was the same as T1, except the successive-number restriction was eliminated; that is, any three different numbers could be chosen.

P5. Randomly selected examples of the numbers 3,5 , and 7 were used on each trial, and their positions in the card holders were determined randomly. All three panel lights were illuminated. Responses to the card that represented the number 7 were reinforced. It may be noted that the illumination of all three panel lights was a consistent cue here and that in succeeding stages responses to the card with the most numerousness entities would be reinforced.

T3. Stimuli were selected in a manner similar to that of T1. However, all panel lights were illuminated, and responses to the card with the most circles were reinforced.

T4. The stimuli were selected as in T2, and the reinforcement contingencies were as in $\mathrm{T} 3$.

T5. Trials similar to those in $\mathrm{T} 1$ and $\mathrm{T} 3$ were presented concurrently. If the center panel light was on, responses to the lowest number were reinforced. If all three panel lights were on, responses to the highest number were reinforced. The order of "low number correct" and "high number correct" was determined randomly, except that each type appeared equally often in two successive 45-trial sessions. The criteria used in this stage of training were to have $41 / 45$ correct overall in a single session and "runs" of $p<.01$ on each of the subsets of "low number correct" and "high number correct" trials.

T6. This was the same as T5, except that the successivenumber restriction was eliminated.

P6. This stage was the same as P4, except that two panel lights, one at each end of the row of three lights, were on, and responses to the card that represented the number 4 were reinforced. It may be noted that the illumination of the two end panel lamps was a consistent cue here and that in succeeding stages responses to the intermediate number would be reinforced.

T7. This stage was the same as T1, except the two end panel lights were on and responses to the intermediate number were reinforced.

T8. This was the same as T7, except that the successivenumber restriction was eliminated. 
T9. Trials like those in $\mathrm{T} 1, \mathrm{~T} 3$, and $\mathrm{T} 7$ were presented concurrently. The condition of the panel lights determined whether responses to the low, intermediate, or high number would be reinforced (i.e., center light only, low; end lights, intermediate; all lights, high). Criteria were a minimum of $13 / 15$ correct on each subset within a session and a "run" of $\mathrm{p}<.01$ on each subset.

T10. T10 was the same as T9, except the successive-number restriction was eliminated.

\section{RESULTS}

One monkey (S 14) met the joint criterion on all numerousness judgment tasks. Another monkey (78-I-2) met criterion on all tasks through $\mathrm{T} 8$. The third monkey (78-I-1) met criterion only through Task T3. Trials to criterion for each monkey on each task completed may be seen in Table 1 .

Training for 78-I-2 was terminated after 16 sessions on Task T9. During the last six sessions, this subject responded on only $22 \%$ of the trials, although he was correct in his responses on $67 \%$ of those trials. Overall on T9, he responded to $59 \%$ of the trials, and he was correct on $75 \%$ of them. A change of incentives (from currants only to random presentations of sunflower seeds, sweet bits of Fruit Loops cereal, and currants) did not increase his rate of responding. He was in good health. His performance was considerably better than chance. It may only be speculated that after nearly 1 year of numerousness judgment training he was insufficiently motivated to perform. It may be recalled that this monkey was used in the Thomas et al. (in press) study just prior to the present one. Although 78-I-1's rate of responding was better than that of 78-I-2, in view of his generally slower progress his training was terminated coincident with that of 78-I-2. His training was terminated after 12 sessions on Task T4 (including 2 in which he refused to make a single response). He

Table 1

Trials to Criterion on Numerousness Pretraining and Training Tasks

\begin{tabular}{crcr} 
& \multicolumn{3}{c}{ Monkeys } \\
\cline { 2 - 4 } Tasks & S14 & $78-\mathrm{I}-1$ & $78-\mathrm{I}-2$ \\
\hline P4 & 270 & 1305 & 405 \\
T1 & 45 & 45 & 180 \\
T2 & 45 & 90 & 270 \\
P5 & 90 & $626^{*}$ & 352 \\
T3 & 45 & 90 & 237 \\
T4 & 45 & & 45 \\
T5 & 90 & & 45 \\
T6 & 180 & & 225 \\
P6 & 45 & & 45 \\
T7 & 90 & & 45 \\
T8 & 90 & & 90 \\
T9 & 270 & & \\
T10 & 45 & & \\
\hline
\end{tabular}

*Any number which is not a multiple of 45 includes sessions in which the monkey would not complete all trials. responded on $92 \%$ of the trials of the remaining 10 sessions, and he had an overall $66 \%$ correct on those trials.

\section{DISCUSSION}

It has been suggested that before attributing conceptual behavior to nonhuman animals the use of cues derived from the specific properties and patterns of the stimuli must have been precluded (e.g., Thomas \& Kerr, 1976). A detailed argument against the use of specific property and pattern cues from the stimuli used in the present study may be found in Thomas et al. (in press). Additionally, in the present work, specific cues could not be used on the relative numerousness tasks to attain criterion, as a stimulus card that was correct on one trial might be one of the incorrect ones on another trial. Thus, it is suggested that the pretraining tasks P4-P6 and the training tasks T1-T10 provided evidence for the conceptual use of numerousness cues by squirrel monkeys.

In addition to the demonstration of the monkeys' abilities to perform conceptual relative numerousness judgments, an important result in the present work was the demonstration that two monkeys were able to judge intermediate numerousness. The ability to distinguish a numerousness set from one more numerous and one less numerous presented at the same time suggests judgments that correspond to the ordinal level of measurement. Thomas and Ingram (1979) demonstrated the squirrel monkey's ability to judge intermediate volume. As noted by them and applicable to the present study as well, whether monkeys are able to show other abilities indicative of "ordinal measurement" (e.g., sorting or rearranging stimuli according to numerousness or size) remains to be demonstrated.

The Brown et al. (1978) comparison of children, chimpanzees, and monkeys on the use of the quantifiers "all," "some," "one," and "none" was conducted in the context that the use of such concepts may be a prerequisite to language. In this regard, the most significant quantifier was "some." As Brown et al. noted, the "all," "one," and "none" tasks might be solved in terms of simple strategies, but "some" could be solved only in terms of a concept of quantity. By implication, the deficiency shown by the apes and monkeys with the concept "some" raises questions either about the ability of apes and monkeys to acquire language or about the prerequisite status of quantitative concepts for the acquisition of language.

Premack (1976), as Brown et al. (1978) noted, had better success in showing his chimpanzee's use of quantitative concepts. Brown et al. also noted that they were unable to explain the discrepancy between their results and those of Premack. Additionally, to the extent that the studies may be compared, the results seen in the present study with intermediate numerousness judgments might appear to be inconsistent with the results of Brown et al. In view of the importance of the question of language and its prerequisites, it may be useful to consider some possible explanations for the differences in the results found by Brown et al. and those reported by Premack and in the present work.

Perhaps the most important difference among the studies was that Brown et al. (1978) required sequential responses from their subjects to denote recognition of the appropriate quantifier. For example, when the concept "some" was relevant, the subject was expected to respond to more than one but not to more than N - 1 of the stimulus objects. In Premack's (1976) study and the present study, the subject made a single response to denote its recognition of the appropriate quantifier. In Premack's case, the animal made a symbolic response; that is, it placed a plastic symbol that represented the quantifier in an appropriate locus. In the present study, the monkey simply displaced the card bearing the set of stimuli representing the concepts "fewest," "most," or "intermediate numerousness." Perhaps the deficiencies reported by Brown et al. were due to 
the more complex response requirement rather than the quantitative judgment per se.

However, another explanation that may account for the Brown et al. (1978) results with the quantifier "some" is concerned with the reinforcement contingencies. In addition to reinforcers' being available beneath the stimulus objects associated with the relevant quantity, the subjects were reinforced also for pressing a lever intended to denote the termination of a trial. Except for the restriction that a leverpress was reinforced only after at least two responses had been made to the objects, the reinforcement contingencies on the "some" trials were such that the subject might obtain all possible reinforcers, regardless of the order in which it emitted its responses. Yet, the trial was considered to be correct only if particular orders of responses were emitted. There was no incentive for the subject to distinguish between correct and incorrect response sequences. One might expect the cues to correct responding associated with the "all," "one," and "none" quantifiers to be more discriminable and, hence, expect to see better performance on those types of trials.

Of course, it should be noted that the children in the Brown et al. (1978) study responded more appropriately to "some" than did the nonhuman primates, and this may point to a question of relevance to language and its prerequisites. Further work is needed to clarify such questions, as well as the distinction between quantitative judgments per se as opposed to the judgment-response relationship.

\section{REFERENCES}

Brown, D. P. F., Lenneberg, E. H., \& Etrtlinger, G. Ability of chimpanzees to respond to symbols of quantity in comparison with that of children and monkeys. Journal of Comparative and Physiological Psychology, 1978, 92, 815-820.

Dooley, G. B., \& GiLl, T. Mathematical capabilities of Lana chimpanzee. In G. H. Bourne (Ed.), Progress in ape research. New York: Academic Press, 1977.

Grant, D. A. Additional tables of the probability of "runs" of correct responses in learning and problem solving. Psychological Bulletin, 1947, 44, 276-279.

HAyes, K. J., \& Nissen, C. H. Higher mental functions of a home-raised chimpanzee. In. A. M. Schrier \& F. Stollnitz (Eds.), Behavior of nonhuman primates: 4. New York: Academic Press, 1971.

Hicks, L. H. An analysis of number-concept formation in the rhesus monkey. Journal of Comparative and Physiological Psychology, 1956, 49, 212-218.

Premack, D. Intelligence in ape and man. Hillsdale, N.J: Erlbaum, 1976.

Salman, D. H. Note on the number conception in animal psychology. British Journal of Psychology, 1943, 33,209-219.

Swenson, L. C. One versus two discrimination by whitenecked ravens (Corvus cryptoleucus) with nonnumber dimensions varied. Animal Behaviour, 1970, 18, 454-460.

Thомas, R. K., \& Crosby, T. N. Absolute versus relative class conceptual behavior in squirrel monkeys (Saimiri sciureus). Animal Learning \& Behavior, 1977, 5, 265-271.

Thomas, R. K., Fowlkes, D., \& Vickery, J. D. Conceptual numerousness judgments by squirrel monkeys. American Journal of Psychology, in press.

Thomas, R. K., \& Ingram, D. K. Conceptual volume judgment by squirrel monkeys. American Journal of Psychology, 1979, 92, 33-43.

Thomas, R. K., \& KeRR, R. S. Conceptual conditional discrimination in Saimiri sciureus. Animal Learning \& Behavior, 1976, 4, 333-336.

Wesley, F. The number concept: A phylogenetic review. Psychological Bulletin, 1961, 53, 420-428.

(Received for publication May 13, 1980.) 